\title{
Traductores españoles de obras científico-técnicas francesas en la revista La Ilustración Española y Americana (1880-1900)
}

\author{
Anna María Corredor Plaja \\ Universitat de Girona \\ annamaria.corredor@udg.edu
}

\begin{abstract}
Resumen: Basándonos en el análisis de los anuncios de obras científicotécnicas traducidas del francés al español, el objetivo de este artículo es presentar un repertorio de los traductores del ámbito científico-técnico del período 1880-1900 que aparecen en la revista, precedidos de una reflexión en torno a la figura del traductor y otros datos de interés. Para ello hemos partido de las informaciones de la sección bibliográfica de la publicación y hemos investigado sobre la identidad de los traductores. ¿Quiénes eran los traductores? ¿Se mencionaban siempre sus nombres? ¿Se hacían alusiones a la calidad de su trabajo? ¿El traductor podía ayudar a "vender" la obra? Estas son algunas de las cuestiones planteadas a las cuales intentamos responder a lo largo del artículo. Palabras clave: traductores científico-técnicos; repertorio; ámbito francésespañol; La Ilustración Española y Americana (1880-1890).
\end{abstract}

\section{Spanish translators of French scientific and technical works in the journal La Ilustración Española y Americana (1880-1900)}

Abstract: Based on the analysis of reviews or little ads from scientific and technical works translated from French to Spanish, the aim of this paper is to present a repertoire of translators of scientific and technical field for the period 1880-1900 appearing in the journal, preceded by a reflection on the figure of the translator and other useful information. So, we have started from the information in the bibliographic section of the publication and we have investigated about the identity of the translators. Who were the translators? Were their names always mentioned? Were allusions to the quality of their work made? Could the translator help "sell" the work? These are some of the issues we try to answer throughout the article.

Keywords: scientific-technical translators; repertoire; French-Spanish field; The Spanish and American Illustration (1880-1890).

\) Corredor Plaja, Anna María. 2016. "Traductores españoles de obras científico-técnicas francesas en la revista La Ilustración Española y Americana (1880-1900)". Quaderns de Filologia: Estudis Lingüistics XXI: 103-125. doi: 10.7203/qfilologia.21.9314 



\section{Presentación}

Como es sabido, La Ilustración Española y Americana (LIEA de ahora en adelante) fue la revista más destacada de la segunda mitad del siglo XIX español. Publicación multidisciplinar y con una clara sensibilidad hacia la cultura extranjera, estamos de acuerdo con Marta Palenque, que la define como "la revista que mejor representa el ambiente cultural de la Restauración" (Palenque, 2013: 15). No sorprende pues que hayamos querido indagar en la sección de bibliografía de esta publicación para estudiar cómo se anunciaban los libros traducidos en nuestro país y, en particular, cómo eran presentados los traductores. La revista se publicó desde 1869 hasta 1921; es obvio que el vaciado de la sección durante estos años supone muchísima información bibliográfica, y que había que delimitar necesariamente el período de estudio. Como explicamos más adelante, en un primer trabajo analizamos globalmente la etapa 1869-1880; para el presente artículo se han analizado los años 1880-1900 y nos hemos centrado solo en las traducciones del ámbito científico-técnico; queda pendiente el período 1900-1921.

A través de su sección de novedades bibliográficas, LIEA puso a disposición de sus lectores anuncios, y en algunos casos auténticas reseñas, de libros nacionales y extranjeros pertenecientes a todas las ramas del saber. Como es sabido, el último tercio del siglo XIX fue en nuestro país uno de los períodos más ricos en cuanto a traducciones, ya que fueron el vehículo de entrada en nuestro territorio de los avances de las ciencias y de la técnica, de las nuevas ideas político-sociales y filosóficas, y de la producción literaria que se desarrollaba en diferentes países europeos.

Según se publicó en el apartado de novedades bibliográficas del primer ejemplar de LIEA (25/12/1869), uno de los objetivos de esta sección fue dar a conocer a sus lectores las obras extranjeras que se traducían a nuestro idioma para que "sirvieran de estímulo" en nuestro país. Es importante destacar que en dicha sección no se diferenciaba entre obras originales y traducciones, en el sentido de que se anunciaban mezcladas. Con este tipo de presentación se pretende mostrar que las traducciones

\footnotetext{
* Este trabajo es fruto de la investigación realizada en el marco del proyecto "Catalogación y estudio de las traducciones literarias en la prensa española (1869-1905)" (Proyecto FFI2009-09561 del Ministerio de Ciencia e Innovación, cofinanciado con fondos FEDER), cuya investigadora principal ha sido la Dra. Marta Giné (UdL).
} 
y los títulos publicados en otros países son equiparables a la producción española y quedan integrados en una misma categoría (Corredor Plaja, 2013). Incluso en una ocasión el reseñador menciona "la competencia" y la "reputación bien adquirida" de los autores, cuando en realidad se está refiriendo a los traductores, como si autor y traductor fueran dos términos sinónimos: es el caso de Lecciones de álgebra elemental y superior, de Charles Auguste Briot, traducido por C. Sebastián y Bernardo Portuendo (08/03/1880: 160). De hecho, mezclar originales y traducciones hasta el punto de crear una cierta confusión en el lector fue una práctica bastante habitual durante el siglo XIX (Botrel, 2010).

El repertorio que presentamos contiene un total de 68 nombres de traductores de obras del ámbito científico-tecnológico: son los que constan como tales en la sección de novedades bibliográficas, con nombre y apellido o con siglas, a los que hay que añadir los que no son mencionados en la sección, donde sus traducciones aparecen como anónimas, pero que hemos localizado en las obras correspondientes. No deja de sorprender que no se haga constar en la revista el nombre del traductor cuando sí figura en el ejemplar traducido, es decir, cuando no ha habido voluntad por parte del traductor de permanecer en el anonimato; desde nuestro punto de vista, en este caso la ocultación del nombre del traductor en el anuncio de la revista debe atribuirse a un puro descuido del reseñador. En cuanto a los traductores que firmaron sus trabajos con siglas, es muy difícil dar con su identidad, y es algo que hemos descartado en el presente trabajo; hemos encontrado tan solo dos traductores que usan siglas, lo cual interpretamos como una estrategia para mantenerse en el anonimato.

\subsection{El tratamiento de la figura del traductor en los anuncios}

Analizando los breves textos de los anuncios, nos damos cuenta de que el nombre del traductor casi siempre se suele indicar junto al del autor y el título de la obra ${ }^{1}$. El nombre del traductor puede aparecer solo, o bien ir acompañado de otras pequeñas informaciones, por ejemplo su pro-

\footnotetext{
${ }^{1}$ La estructura que presentan los textos de la sección bibliográfica durante el período 1869-1880 fue analizada con detalle en el trabajo "Presencia de la traducción en las novedades bibliográficas de La Ilustración Española y Americana (1869-1880)" (Corredor Plaja, 2012). No insistiremos aquí sobre este aspecto, dado que se mantuvo la misma estructura en los años posteriores.
} 
fesión y/o el cargo que ocupa. En este último caso, puede interpretarse esta información como una garantía de la calidad del texto, tal como señala Jean-René Aymes: "En casi todos los casos, son sus cargos, su carrera anterior o sus títulos lo que les designa como traductores adecuados y, por tanto, recomendables" (Aymes, 2010: 90).

En efecto, que el Manual práctico de oftalmología, de Georges Camuset, fuera traducido por el oftalmólogo Peregrín Bayarri, que además fue autor de al menos dos obras del campo de la medicina, es algo que inspira confianza; que Vicente Peset y Cervera, cirujano y químico, y autor de varias obras del ámbito de la medicina y la farmacología, tradujera el Tratado de química biológica, de Charles AdolpheWurtz, es una garantía de que se ofrece al lector una buena traducción. Es cierto que no conocemos el dominio de la lengua de partida que tenían estos traductores, pero aun así, la afinidad entre sus especialidades y las materias traducidas son un indicio importante de la calidad de su trabajo. Como afirma Eusebio Martínez de Velasco (V.) a propósito de la traducción de Higiene de la vista, de Alexandre Magne, realizada por Casiano Macías y Rodríguez, oftalmólogo: "Nadie mejor que un médico especialista podía verter a nuestro idioma una obra tan importante como necesaria, para que esté correcta" (LIEA, 30/08/1881: 95).

Como puede observarse en el repertorio, hay otros muchos casos en los que se produce esta afinidad. Pero también hay algún ejemplo en el sentido contrario: así, Bernardo Portuondo y C. Sebastián figuran en LIEA como "comandantes de artillería e ingenieros del ejército y exprofesores de las academias especiales de sus armas respectivas" y tradujeron Lecciones de álgebra elemental y superior, de Charles Auguste Briot; asimismo, José Muñoz Maldonado, vizconde de San Javier, periodista, novelista, historiador, jurista, político, tradujo La hidráulica, de E. Marzy. Igualmente, llama la atención el caso de Mariano Blanch, traductor de unas cuantas obras de Victor Hugo, pero también de Fisiología descriptiva de las treinta bellezas de la mujer, del fisiólogo Auguste Debay.

\subsection{Las lenguas de los originales}

En la reseña se suele indicar la edición y la lengua de la que se traduce, pero hay alguna excepción. Por ejemplo, en el anuncio siguiente no se indica la lengua de partida: 
Tratado práctico de las enfermedades de la garganta y de las fosas nasales por el Dr. E. F. Moure, traducido y anotado extensamente por el Dr. Celestino Compaired (LIEA, 30/07/1896).

Investigando un poco, hemos encontrado que Moure (cuyas iniciales eran E. J., Émile-Jean, y no E. F., como aparece en la revista) no era el autor de esta obra, tal como figura en la reseña y en el ejemplar español, sino que junto con un tal J. Charazac la habían traducido del inglés al francés y el verdadero autor era Sir Morell Mackenzie, un prestigioso laringólogo inglés, cuyo nombre aparece en la traducción francesa pero no en la española, que en realidad es una traducción indirecta del inglés. Dado que utilizar el francés como lengua intermedia era una práctica bastante frecuente en el siglo XIX (Zaro, 2008), es muy posible que en el repertorio haya otros casos de traducciones indirectas, pero no era el propósito de este trabajo indagar en este sentido. Además, hay que tener en cuenta que, cuando se trata de autores menores y de obras poco relevantes, descubrir la lengua del original puede convertirse en una auténtica odisea, cuando no en algo imposible.

Señalemos que solo en algunos casos sí se precisa que se trata de traducciones directas. Cuando no se da esta indicación, como en el ejemplo anterior, no podemos saber si estamos ante una traducción directa o indirecta. Desde el punto de vista del lector de la época, que lo único que quería era acceder al texto en lengua española, es evidente que este detalle debía de ser una cuestión menor.

\subsection{Los complementos de las traducciones}

Otro dato que suele constar en la reseña es si la traducción contiene un prólogo o si el traductor ha hecho algo más que dedicarse a traducir el original, como si este algo más fuera un mérito añadido a la traducción. Como indican Julia Pinilla y Brigitte Lépinette $(2009)^{2}$, los traductores no se limitaban a traducir sino que adaptaban la obra a sus receptores, asumiendo así el papel de "intermediarios culturales" en su afán de divulgar los conocimientos extranjeros en nuestro país. Así, en uno de los

\footnotetext{
${ }^{2}$ Aunque el estudio de Pinilla y Lépinette está centrado en el período de la Ilustración, sus observaciones son perfectamente válidas para las traducciones realizadas a lo largo del siglo XIX.
} 
anuncios de la versión española del Diccionario de medicina y cirugía, farmacia, veterinaria y ciencias auxiliares, de Émile Littré, leemos:

Versión española de la decimoquinta edición francesa, aumentada y puesta al corriente de las ciencias médicas y biológicas, y de la práctica diaria, por los doctores J. Aguilar Lara y M. Carreras Sanchís, y precedida de un prólogo del Dr. Amalio Jimeno (LIEA, 15/10/1893: 238).

En este caso, se ha añadido un prólogo, y por "aumentada y puesta al corriente" podemos interpretar que, como mínimo, se han añadido entradas, o acepciones, o se han ampliado las definiciones con el fin de actualizarlas. Otro ejemplo curioso es el de la traducción del Manual de higiene pública y privada, de Armand B. Paulier, que en uno de los anuncios aparece simplemente traducido por Álvaro Arnau y Clemente, con el comentario "que recomendamos" (15/02/1881), mientras que en un anuncio anterior y en otro posterior se indica que está anotado por el doctor Constantino Gómez. En otras ocasiones, no queda muy claro lo que se añade; por ejemplo, en la reseña del Nuevo y completo manual del mejoramiento de los líquidos tales como: vinos, vinos espumosos, alcoholes..., de Valentin Ferdinand Lebeuf, traducido por J. Calva y De Grassot, leemos: "tercera edición, traducida al castellano por D. J. Calva y de Grasot, y aumentada con el arte de fabricar los vinos artificiales en las colonias" (LIEA, 15/03/1883: 166); o también en el anuncio de Tratado de medicina legal de jurisprudencia médica y de toxicología, de Legrand du Saulle et al., traducido por Teodoro Yáñez y Font y Carlos Núñez y Granés, donde se hace constar: "Traducido, anotado y aumentado con la legislación médico-legal española, la inglesa, y la de las diferentes repúblicas americanas, comparada y comentada..." (LIEA, 22/04/1887: 270).

Entendemos que este "paratexto del traductor", expresión que tomamos de Pinilla y Lépinette (ibid.), puede llegar en algunas ocasiones a modificar substancialmente la obra original. Veamos algunos ejemplos: en el anuncio del Tratado elemental de química, de L. Troost, traducido por Eduardo Cazorla, el reseñador señala:

Esta nueva traducción del señor Cazorla difiere esencialmente de la anterior, por las numerosas adiciones hechas al texto, especialmente en el desarrollo de los principios de la termoquímica, así como en su modo y claridad (LIEA, 22/03/1890: 190). 
Igualmente, se presenta la obra Vocabulario de términos de arte, de Jules Adeline, traducida por José Ramón Mélida, en estos términos: "traducida al castellano, y aumentada con gran número de voces concernientes a las Artes y su tecnicismo" (LIEA, 30/11/1888: 223). En definitiva, estos añadidos (prólogos, apéndices, anotaciones, etc.) son muy frecuentes y se perciben como algo positivo, que enriquece el texto original y facilita la comprensión al lector destinatario.

\subsection{La crítica de los reseñadores}

En algunas ocasiones, el reseñador hace un breve comentario sobre la calidad de la traducción, con expresiones como: "la versión castellana está bien hecha", "la traducción está muy bien hecha", "la traducción se ha verificado también con gran esmero", "nuevo y excelente trabajo literario" (aunque no se trate de ninguna obra literaria), o bien menciona "los vastísimos conocimientos" que el traductor posee de la materia, o bien recomienda la obra a un público determinado, con comentarios del tipo: "Es una obra de imprescindible necesidad para..." (médicos, militares, madres de familia, alumnos de tal especialidad, artistas, etc.). En una ocasión, en el anuncio de El arte de vivir, tratado completo de higiene, de Hubert Boëns, traducido por Rafael Fernández Esnaola, consta que la versión española ha sido hecha "con autorización del autor", como si esto fuera una garantía de la calidad del texto.

Los comentarios de los reseñadores son casi siempre positivos y contribuyen a publicitar las traducciones. Como curiosidad señalamos que en más de una ocasión es el propio traductor quien gestiona la venta de ejemplares, por lo que en la misma reseña se indica su domicilio para que el lector encargue directamente los pedidos. Es el caso de Pablo Álvarez Delgado, traductor de Tratamiento de la agonía, de Hubert Boëns, o de Manuel Flores y Pla, traductor de Lecciones sobre las enfermedades del sistema nervioso, de Jean-Martin Charcot, ambos domiciliados en Madrid.

\section{Repertorio de traductores}

Presentamos a continuación el listado de traductores. Hemos hecho una entrada para cada uno de ellos, ordenados alfabéticamente. En primer lugar aparece cada nombre completado, cuando ha sido posible, con 
algunos datos sobre las actividades por las que el traductor es conocido. En ocasiones no ha sido fácil encontrar dicha información: que el nombre o uno de los apellidos del traductor aparezca en la revista con una simple inicial, que el autor de la traducción no fuera una persona conocida, que utilizara siglas, son complicaciones que dificultan la búsqueda. Hay que señalar que del total de 68 traductores con entrada en el repertorio hay 21 sobre los que no hemos podido localizar ninguna información. En segundo lugar, anotamos las obras traducidas y sus autores (T), así como su localización en LIEA (fecha y página del ejemplar). Asimismo hemos considerado oportuno añadir, cuando ha sido posible hallarlos, los datos de los originales traducidos $(\mathrm{O})$ : el título original y lugar, editorial y fecha de la primera edición.

AGUILAR LARA, Juan. Profesor clínico en la Escuela de Medicina de Valencia y autor de varias obras del ámbito de la medicina.

T: Diccionario de medicina y cirugía, farmacia, veterinaria y ciencias auxiliares (traducción compartida con Manuel Carreras Sanchís), de Émile Littré y Charles-Philippe Robin (15/03/1888: 181 y ss.).

O: Dictionnaire de médecine, de chirurgie, de pharmacie, des sciences accessoires et de l'art vétérinaire. Paris: Baillière, 1855.

AGUIRRE, Antonio

T: Enciclopedia de construcción (traducción anónima en LIEA) de L. A. Barré (15/02/1899: 99).

O: Petite encyclopédie pratique du bâtiment. Paris: Bernard, 1898.

ÁlVAREZ DELGADO, Pablo

T: Tratamiento de la agonía, de Hubert Boëns (15/06/1883: 304).

ÁLVAREZ SEREIX, Rafael (Madrid, 1855-1946). Ingeniero de Montes, político, escritor, traductor y conferenciante. Autor de numerosas obras.

T: Origen y desarrollo de la vida en el globo, del marqués de Nadaillac (30/07/1889: 67); La ciencia y el materialismo, de Jules-ErnestNaville (30/08/1891: 128).

$\mathrm{O}$ : L'origine et le développement de la vie sur le globe, Paris: De Soye\&Fils, 1888; La science et le matérialisme: étude philosophique. Genève: A. Cherbuliez, 1891. 
ANGERS, Armando

T: El fonógrafo Edison y sus aplicaciones, de A.-MathieuVillon, (15/12/1896: 351).

O: Le phonographe et ses applications. Paris: B. Tignol, 1894.

ANGULO Y SUERO, Francisco. Coautor de Fitografía y flórula farmacéutica hispánica (1885).

T: Tratado de toxicología y de química legal aplicada a los envenenamientos, de Joseph-Théodore-Pierre Chandelon (30/10/1889: 262).

O: Traité de toxicologie et de chimie légale appliquée aux empoisonnements. Liège: H. Vaillant-Carmanne, 1888.

ARNAU Y CLEMENTE, Álvaro. Médico y cirujano.

T: Manual de higiene pública y privada, de Armand B. Paulier (22/12/1880: 384, y ss.).

O: Manuel d'hygiène publique et privée. Paris: Doin, 1879.

BAYARRI, Peregrín. Oftalmólogo, autor de al menos dos obras del campo de la medicina.

T: Manual práctico de oftalmología, de Georges Camuset (08/02/1888: 104).

O: Manuel d'Ophtalmologie. Paris: Masson, 1877.

BLANCH, Mariano. En 1879 viajó a París con el editor José Espasa, donde compraron algunas obras para traducir al español.

T: Fisiología descriptiva de las treinta bellezas de la mujer, de Auguste Debay (15/07/1880: 32).

O: Physiologie descriptive des trente beautés de la femme. Paris: Dentu, 1879.

BLANCO VÁZQUEZ, Eduardo. Médico.

T: Higiene y saneamiento de las poblaciones, de Jean Baptiste Fonssagrives (08/08/1885: 80).

O: Hygiène et assainissement des villes, Paris: Baillière, 1874.

CALVA Y DE GRASSOT, J.

T: Nuevo y completo manual del mejoramiento de los líquidos tales como: vinos, vinos espumosos, alcoholes..., de Valentin Ferdinand Lebeuf (15/03/1883: 166). 
O: Nouveau manuel complet de l'amélioration des liquides tels que vins, vins mousseux, alcools, eaux-de-vie, liqueurs, kirschs, rhums, cidres, vinaigres, etc. Paris: Roret, 1862.

CARILLA, D. H. Médico.

T: Tratado de las enfermedades del estómago, de VictorAudhoui (22/08/1886: 112).

O: Traité des maladies de l'estomac. Paris: Delahaye et Lecrosnier, 1883.

CARRERAS SANCHÍS, Manuel. Médico.

T: Estudio sobre la anestesia quirúrgica (traducción anónima en LIEA), de H. de Brinon (08/07/1883: 16); Enciclopedia de terapéutica general (traducción compartida con Celestino Compaired y Cabodevi1la), de J. Bauer (et al.) (15/06/1888 y ss.).

O: Traité d'anesthésie chirurgicale. 1880.

Véase también: Aguilar Lara, Juan.

CASES CIVERA, Ramon

T: Guía práctica de electricidad industrial (traducción anónima en LIEA), de Georges Dumont y Gustave Baignères (08/07/1896: 15); Pequeña enciclopedia electro-mecánica (traducción compartida con León Soulerat, pero anónima en LIEA), de Henri de Graffigny (15/11/1896: 287 y ss.).

O: Guide pratique d'électricité industrielle. Paris: Paul Dupont, 1894; Petite encyclopédie électro-mécanique. Paris: Bernard, 1896.

CAZORLA, Eduardo

T: Tratado elemental de química, de LouisTroost. (22/03/1890: 190).

O: Traité élémentaire de chimie. Paris: Masson, 1869.

CODINA CASTELLVÍ, José (Reus, 1867-Madrid, 1934): médico, miembro numerario de la Real Academia Nacional de Medicina. Autor de varios libros del ámbito de la medicina.

T: Elementos generales de farmacodinamia, de Diomède de Buck (15/10/1894: 231). 
COLVÉE ROURA, Pablo (Elbeuf, 1849-Valencia, 1903). Médico y químico, destacó en el campo de la microbiología. Académico de la Real Academia de Medicina de Valencia.

T: Manual de patología interna, escrito para uso de profesores y estudiantes, de Constant François Vanlair (22/11/1893: 328, y ss.).

O: Manuel de pathologie interne à l'usage des étudiants et des praticiens. Liège: Desoer, 1890.

COMPAIRED Y CABODEVILLA, Celestino (Erla, 1858-Madrid, 1942). Médico otorrino. Autor de obras del ámbito de la medicina.

T: Tratado práctico de las enfermedades de la garganta y de las fosas nasales, de Morell Mackenzie (30/07/1896: 64, y ss.).

O: Traité pratique des maladies du nez et de la cavité naso-pharyngienne. Paris: OctaveDoin, 1887. (Traducido del inglés: A Manual of the Diseases of the Nose and Throat).

Véase también: Carreras Sanchís, Manuel.

COROMINAS Y SABATER, José (?, 1850-?, 1907). Médico, autor de varias obras del ámbito de la medicina.

T: Tratado de anatomía topográfica, de Paul Jules Tillaux (22/09/1880: 184).

O: Traitéd'anatomie topographique avec applications à la chirurgie. Paris: Asselin, 1872.

C. S. y P.

T: Los microbios, de FolHermann (08/10/1885: 216).

O: Les microbes. Genève: Georg, 1885.

DÉLANO, Manuel A. Autor de varios libros, especialmente del ámbito de la química.

T: Exposizión elemental de los prinzipios fundamentales de la teoría atómica [sic], de Georges Denigès (22/04/1893: 276).

O: Exposé élémentaire des principes fondamentaux de la théorie atomique. Paris: Masson, 1892.

DEÓ, Manuel

T: Tratado del cultivo de la vid y vinificación, de Jules Guyot (15/01/1882: 46, y ss.); Tratados de la posesión y prescripción, de Robert-Joseph Pothier (15/07/1880: 32, y ss.). 
O: Culture de la vigne et vinification. Paris: Librairie agricole de la Maison rustique, 1860; Traité de la possession et de la prescription. Paris: Debure père, 1772.

ESCRIBANO GARCÍA, Eduardo (En LIEA aparece como Eduardo E. García). Fundador del periódico La Irradiación (1892), editor de una Revista Bibliográfica Universal (1908) y secretario de la revista La fraternidad universal. Escribió varias obras del ámbito del espiritismo.

T: ¿Qué es el cielo?, de Camille Flammarion (08/10/1896: 208)

O: Qu'est-ce que le ciel? Paris: Marpon et Flammarion, 1892.

FERNÁNDEZ ESNAOLA, Rafael. Codirector de la revista La Medicina Rural, que empezó a publicarse en Madrid en diciembre de 1881.

T: El arte de vivir, tratado completo de higiene, de Hubert Boëns (15/05/1882: 311).

O: L'art de vivre. Traité complet d'hygiène et de médecine à l'usage des gens du monde. Bruxelles: F. Hayez.

\section{FLORES Y PLA, Manuel}

T: Lecciones sobre las enfermedades del sistema nervioso, de JeanMartin Charcot. (15/04/1883: 238); Tratado de la higiene de la infancia, de Jean Baptiste Fonssagrives (15/04/1885: 232, y ss.).

O: Leçons sur les maladies du système nerveux. Paris: Delahaye, 1872; Leçons d'hygiène infantile. Paris: Delahaye et Lecrosnier, 1882.

FUENTE, Federico de la. Profesor de electrotecnia de la Escuela Central de Artes y Oficios de Madrid.

T: Los acumuladores eléctricos, de Jules-Armand Montpellier (08/06/1897: 351).

O: Les accumulateurs électriques: montage, installation, conduite, entretien. Paris: H. Desforges, 1896.

GILI Y ROIG, Gustavo (Irún, 1868-Barcelona, 1945). Editor y político, fundador de la editorial Gustavo Gili (1902).

T: Enfermedades agudas y crónicas, de Nicholas Neuens (30/05/1895: 348).

O: Traitement naturel des maladies aiguës et chroniques d'après le système Kneipp. Paris: P. Lethielleux, 1895. 
GUERRERO, Vladimir. Ingeniero agrónomo, miembro de la Sociedad Química de París.

T: Tratado del cultivo de la remolacha azucarera, de Georges Dureau (15/12/1891: 382).

O: Traité de la culture de la betterave à sucre. Paris: Bureaux du Journal des Fabricants de Sucre, 1886.

HIDALGO MOBELLÁN, Antonio. Miembro de la Sociedad de Geografía de México.

T: Las instalaciones de alumbrado eléctrico, de G. Fournier y J. A. Montpellier (22/08/1892: 115).

O: Les installations d'éclairage électrique. Paris. Georges Carré, 1890.

JANINI, Rafael (Tarragona, 1866-Valencia, 1948). Ingeniero agrónomo, destacó en el campo de la enología.

T: Las enfermedades de la vid, de Pierre Viala (22/06/1891: 392).

O: Les maladies de la vigne. Montpellier: Camille Coulet / Paris: Delahaye et Lecrosnier, 1885.

JIMENO CABAÑAS, Antonio. Capitán graduado, teniente del regimiento de caballería de Sagunto.

T: Curso de topografía militar, de Émile-Eugène Poirot (08/07/1880: 16).

O: Cours d'études militaires: cours de topographie destiné... Paris: Baudoin et Cie, 1878.

LÓPEZ DÍAZ, José. Primer profesor del Instituto Oftálmico de Madrid.

T: Tratado elemental de patología externa (traducción compartida con Mariano Salazar y Alegret, y E. Santana y Villanueva), de EugèneFollin y Simon E. Duplay (22/02/1882: 127, y ss.).

O: Traité élémentaire de pathologie externe. Paris: Masson, 1869.

LLANZA, Eugenio de (E. de LL. en LIEA)

T: Manual práctico para el análisis de los vinos, de J. T. V. Muller (30/01/1883: 72); Fabricación de vinos espumosos, de L. Jaunay y Edme-Jules Maumené (30/01/1883: 72); El arte de hacer el vino, de Claude Ladrey (15/10/1884: 230). 
O: Indications théoriques et pratiques sur le travail des vins et en particulier sur celui des vins mousseux. Paris: Masson, 1858; L'art de faire le vin. Paris: F. Savy, 1863.

LLAURA, D. E.

T: Fabricación de vinos de frutas y bebidas económicas, de Nicolas Basset (30/03/1883: 208).

$\mathrm{O}$ : es posible que se trate de: Guide théorique et pratique du fabricant d'alcools et $d u$ distillateur. Paris: Librairie du Dictionnaire des arts et manufactures, 1868.

MACÍAS Y RODRÍGUEZ, Casiano. Oftalmólogo.

T: Higiene de la vista, d'Alexandre Magne (30/08/1881: 95).

O: Hygiène de la vue. Paris: Baillière, 1847.

MARCH, Eduardo

T: Viajes aéreos, de Camille Flammarion (30/05/1881: 363).

O: Mes voyages aériens. Paris: Marpon et Flammarion, 1883.

MARTÍNEZ RUIZ DE LINARES, Antonio (Antonio M. Ruiz de Linares en $L I E A$ ). Primer teniente de infantería.

T: Estudios de táctica y examen de las consecuencias de la adopción de las armas de pequeño calibre..., de Alexandre-Charles Luzeux (08/06/1891: 360, y ss.).

O: Études de tactique et examen des conséquences de l'adoption des armes de petit calibre, à tir rapide, et de la poudre sans fumée. Paris: Baudoin, 1890.

MÉLIDA, José Ramón (Madrid, 1856-1833). Destacado arqueólogo y autor de diversas obras sobre arqueología e historia del arte, además de novelista y dramaturgo. Fue docente en la Universidad Central de Madrid y desempeñó cargos en diferentes instituciones (fue director del Museo Arqueológico Nacional).

T: Vocabulario de términos de arte, de Jules Adeline (30/11/1888: 223).

O: Lexique des termes d'art. Paris: Quantin, 1884. 
M. P. O.

T: Guía teórico-práctica para combatir las enfermedades de la vid, de J. T. V. Muller (08/09/1882: 142).

MUÑOZ MALDONADO, José (vizconde de San Javier en LIEA) (Alicante, 1807-1875). Conde de Fabraquer. Historiador, jurista, periodista y novelista, ministro del Tribunal Supremo de Guerra y Marina. Dirigió el Semanario Pintoresco Español, El Museo de las Familias, El Mentor de la Infancia, El Domingo y Flor de la Infancia. Autor de diversas obras.

T: La hidráulica, de E. Marzy (15/08/1880: 96).

O: L'hydraulique. Paris: Hachette, 1868.

NAVARRO, Francisco (puede tratarse de Francisco Navarro Villoslada) (Viana, 1818-1895). Periodista, político, novelista, poeta y traductor.

T: El cultivo de la viña y los abonos químicos, de Henri Joulie (H. Foulié en LIEA). (22/01/1880: 56).

O: La culture de la vigne et les engrais chimiques. Paris/Bordeaux: Société anonyme des produits chimiques agricoles, 1876.

NEIRA Y CAYLLET, José de. Médico, miembro de la Liga Internacional de Antivacunistas.

T: véase Ruiz y Olavarria, Narciso.

NÚÑEZ GRANÉS, Carlos (Benavente, 1856-1902). Médico, abogado y diputado.

T: véase Yáñez y Font, Teodoro.

OCHOA, Eugenio de (Lezo, 1815-Madrid, 1872). Traductor, poeta, novelista, editor, articulista, dramaturgo y crítico teatral. Figura destacada del Romanticismo español.

T: Enciclopedia del fotógrafo aficionado, de Georges Brunell (08/10/1900: 211).

O: Encyclopédie de l'amateur photographe. Paris: Tignol, 1897.

OLÓRIZ AGUILERA, Federico (Granada, 1855-Madrid, 1912). Médico y antropólogo. 
T: Resumen de anatomía patológica, seguido de un apéndice con indicaciones técnicas para la práctica de las autopsias, de Louis Bard (08/02/1891: 86); La antropología criminal (traducción compartida con Jerónimo Vida), de Xavier Francotte (22/05/1893: 346-348).

O: Précis d'anatomie pathologique. Paris: Masson, 1899; L'anthropologi e criminelle. Paris: Baillière, 1891.

ORELLANA, E. J.

T: Tratado de la fabricación de licores de todas clases, de L.-F. Dubief (30/05/1880: 360).

O: es posible que se trate de: Traité de la fabrication des liqueurs françaises et étrangères, sans distillation. Paris: Lacroix, 1879.

\section{PALANCA Y LITA, Ricardo}

T: Curso completo de prestidigitación y hechicería antigua y moderna, de Jean-NicolasPonsin (30/12/1881: 399, y ss.); Arte de ganar a todos los juegos, de Jean-Eugène Robert-Houdin (08/05/1880: 304); Los secretos de la prestidigitación y de la magia, de Jean-Eugène Robert-Houdin (08/09/1889: 142).

O: La sorcellerie ancienne et moderne expliquée ou cours complet de prestidigitation. Paris: Roret, 1853; L'art de gagneràtous les jeux. Tricheries des Grecs dévoilées. Paris: Calmann-Lévy, 1861; Les secrets de la prestidigitation et de la magie. Comment on devient sorcier. Paris: Lévy Frères, 1868.

PÉREZ DE VILLAOZ, José María

T: El arte de colorear los vinos, del Dr. Prunaire (15/09/1881: 160, y ss.).

O: L'art décolorer les vins avec la couleur naturelle du raisin. Lyon: Bourgeon, 1877.

PESET Y CERVERA, Vicente (Valencia, 1855-1945). Médico cirujano y químico, ejerció docencia en la Facultad de Medicina de la Universidad de Valencia. Autor de varias obras del ámbito de la medicina y la farmacología.

T: Tratado de química biológica, de Charles Adolphe Wurtz (22/12/1890: 392, y ss.).

O: Traité de chimie biologique. Paris: Masson, 1885. 
PLAZA Y CASTAÑOS, José. Médico y escritor, fue profesor en el Hospital General de Madrid y ejerció cargos en el ámbito de la sanidad. T: La sugestión y sus aplicaciones terapéuticas, de Samuel Bernheim (22/11/1886: 302).

O: De la suggestion et de ses applications à la thérapeutique. Paris: Octave Doin, 1888.

PORTUONDO Y BARCELÓ, Bernardo. Autor de Lecciones de arquitectura. Profesor de la Academia de Ingenieros de Madrid, teniente coronel del ejército.

T: véase Sebastián, C.

PRIETO, M.

T: Estudios sobre el vinagre, de Louis Pasteur (22/11/1882: 312).

$\mathrm{O}$ : Études sur le vinaigre, sa fabrication, ses maladies, moyens de les prévenir. Paris: Gauthier-Villars, 1868.

PUIGFERRER, Joaquín. Se trata de Joaquim Puigferrer i Figueras. Médico cirujano, defensor de la medicina popular, tradujo al castellano las obras médicas y científicas de François-Vincent Raspail.

T: Novísimo manual de la salud (traducción anónima en LIEA), de François-Vincent Raspail (15/09/1882: 160).

O: Manuel annuaire de la santé ou médecine et pharmacie domestiques. Paris: Raspail/Lyon: Charles Savy, 1882.

RUBIO, Ricardo. Secretario del Museo Pedagógico Nacional.

T: El ejercicio en los adultos, de Fernand Lagrange (08/03/1896: 151); La evolución de las ideas generales, de Théodule Ribot (15/01/1900: 36); La herencia psicológica, de Théodule Ribot (08/05/1900: 275); La psicología de los sentimientos, de Théodule Ribot (30/09/1900: 196); La sugestión: su función educativa, de Félix Thomas (30/06/1900: 388).

O: De l'exercice chez les adultes. Paris: Alcan, 1891; L'évolution des idées générales. Paris: Alcan, 1897; L’hérédité. Étude psychologique. Paris, 1873; La psychologie des sentiments. Paris: Alcan, 1896; La suggestion: son rôle dans l'éducation. Paris: Alcan, 1895. 
RUIZ Y OLAVARRÍA, Narciso (Nicasio en LIEA). Médico, miembro de la Liga internacional de antivacunistas.

T: No más vacunación, no más vacuna (traducción compartida con José de Neira y Cayllet), de HubertBoëns (15/06/1883: 374, y ss.).

O: es posible que se trate de: La vaccine au point de vue historique et scientifique. Travaux originaires du Congrès de Cologne en octobre 1881; correspondances, mémoires, discussions. Charleroi: Piette, 1882.

SALAZAR Y ALEGRET, Mariano (Barcelona, 1843-1896). Médico, profesor del Hospital de la Princesa y miembro numerario de la Real Academia de Medicina.

T: Véase López Díaz, José.

SAN ROMÁN, Antonio de. Ingeniero de minas.

T: Diccionario de electricidad y magnetismo y sus aplicaciones a las ciencias, las artes y la industria, de Julien Lefèvre (15/06/1893: 400, y ss.).

O: Dictionnaire d'électricité et de magnétisme, contenant les applications aux sciences, aux arts et à l'industrie. Paris: Baillière, 1891.

SANTANA Y VILLANUEVA, Francisco. Profesor clínico de la Facultad de Medicina de la Universidad Central de Madrid.

T: Véase López Díaz, José.

SANTERO, Francisco Javier. Ejerció docencia en la Facultad de Medicina de la Universidad Central de Madrid y fue miembro de la Real Academia de Medicina.

T: Lecciones de clínica médica, de Sigismond Jaccoud (30/06/1888: 421 , y ss.).

O: Leçons de clinique médicale. Paris: Delahaye, 1867.

SEBASTIÁN, C. Junto con Bernardo Portuendo figuran en el anuncio como "comandantes de artillería e ingenieros del ejército y ex-profesores de las academias especiales de sus armas respectivas".

$\mathrm{T}$ : Lecciones de álgebra elemental y superior (traducción compartida con Bernardo Portuendo), de Charles Auguste Briot (08/03/1880: 160). 
O: es posible que se trate de: Leçons d'algèbre conformes aux programmes officiels de l'enseignement des lycées. Paris: Dunod, 1863.

\section{SOULERAT, León}

T: Manual del electroquímico (traducción anónima en LIEA), de Henri de Graffiny (30/10/1897: 267-268); La electricidad para todos (traducción anónima en LIEA), de Henri de Graffiny (30/10/1897: 267-268).

O: Guide pratique de l'électro-chimiste. Paris: Bernard et Cie, 1896; L'électricité pour tous. Paris: Bernard et Cie, 1896.

Véase también: Cases Civera, Ramon.

TANIR, M. A.

T: Magia y física recreativa, de Jean-Eugène Robert-Houdin, (15/08/1890: 95).

O: Magie et physique amusante. Paris: Calmann-Lévy, 1877.

\section{TOLEDO, Federico}

T: Anuario de terapéutica, materia médica, farmacia e higiene (traducción compartida con Rafael Ulecia), de Apollinaire Bouchardat (30/05/1880: 360).

O: Annuaire de thérapeutique, de matière médicale, de pharmacie et d'hygiène. Paris, 1879.

ULECIA, Rafael (se trata de Rafael Ulecia y Cardona, a veces firma con un solo apellido) (Santiago de Cuba, 1850-Madrid, 1912). Médico pediatra, fundador de la Revista de Medicina y Cirugía Prácticas y los Anales de Obstetricia, Ginecopatía y Pediatría. Muy popular por haber creado la institución "La Gota de Leche".

T: Del uso de los baños de mar en los niños, de André-ThéodoreBrochard (15/08/1881: 95); Primeros auxilios a los envenenados, a los ahogados, a los asfixiados, a los heridos en caso de accidente y a los enfermos en caso de indisposición repentina, de Eusèbe Ferrand (22/06/1887: 406); El nicotinismo, de Émile Laurent (08/02/1894: 92).

O: Des bains de mer chez les enfants. Paris: Baillière, 1864; Premiers secours aux empoisonnés, aux noyés, aux asfixiés, aux blessés en 
cas d'accident et... en cas d'indisposition subite. Paris: Baillière, 1878.

Véase también: Toledo, Federico.

VALLE Y ALDABALDE, Rafael del (Gijón, 1857-Madrid, 1936). Médico, asumió durante dos años el cargo de presidente de la Academia Médico-Quirúrgica Española y fue académico de número de la Real Academia Nacional de Medicina. Es autor de numerosas publicaciones y tradujo del francés y del alemán.

T: Los microbios patógenos, de Charles Jacques Bouchard (28/02/1893: 143).

O: Les microbes pathogènes. Paris: Baillière, 1892.

VIDA, Jerónimo

T: véase Olóriz Aguilera, Federico.

\section{VILLALBA Y RIQUELME, Ricardo}

T: La tracción eléctrica, de C. Tainturier (15/01/1898: 36).

O: La traction électrique. Paris: Librairie Industrielle Fristel, 1897.

YÁÑEZ Y FONT, Teodoro (Barcelona, ?-Madrid, 1902). Ejerció docencia en la Facultad de Medicina de la Universidad Central de Madrid. Fue redactor de la revista El Pabellón Médico.

T: Tratado de medicina legal de jurisprudencia médica y de toxicología (traducción compartida con Carlos Núñez y Granés), de Legrand du Saulle et al. (22/04/1887: 270).

O: Traité de médecine légale, de jurisprudence médicale et de toxicologie. Paris Delahaye et Lecrosnier, 1886.

El repertorio de traductores permite corroborar que en la mayoría de las ocasiones existe una afinidad entre la formación o la dedicación del traductor y la materia que traduce, que, como hemos indicado anteriormente, es un indicio a tener en cuenta a la hora de valorar la calidad de una traducción. Un análisis rápido de los títulos (traducidos u originales) permite comprobar la presencia de unas cuantas palabras recurrentes; así, por ejemplo "vid" o "vino" (singular o plural) aparecen en nueve títulos, y los términos "higiene", "enfermedades" y "electricidad" (o derivados) forman parte de seis títulos: es un dato significativo 
en la medida en que las nuevas publicaciones representan la evolución de la sociedad que las genera. En estos casos en concreto, los títulos son, por así decirlo, un eco del desastre causado por la filoxera, pero también del auge de la fabricación de los vinos y licores, de los avances de la medicina en el estudio de diferentes patologías, de la importancia de la higiene en la prevención de las enfermedades o en el cuidado de la imagen personal, de las aplicaciones de la electricidad en diferentes campos de la tecnología.

Otro aspecto que permite observar el repertorio es el período de tiempo que separa la publicación del original de la publicación de la traducción; en algunos casos, ambas publicaciones son casi simultáneas, ya que la traducción aparece publicada el mismo año que el original. Desde luego sería necesaria una investigación específica para determinar en qué casos existían traducciones anteriores a las que se recogen en este trabajo o a partir de qué edición del original se realizó la traducción española. Pero el análisis en profundidad tanto de los títulos (¿cómo han sido traducidos los títulos originales?) como de las condiciones de publicación son aspectos que no habíamos contemplado al plantearnos el objetivo del trabajo, centrado, como hemos indicado al principio del artículo, en el tratamiento de la figura del traductor en los textos de la sección de novedades bibliográficas de LIEA.

\section{Conclusiones}

Retomando las preguntas formuladas al iniciar el trabajo, podemos concluir lo siguiente: en la mayoría de las ocasiones el nombre del traductor consta en la reseña, pero en algún caso, no aparece a pesar de figurar en el ejemplar traducido, lo cual podemos atribuir a un descuido del reseñador o, por qué no, a su deseo de dar prioridad al título y al autor. Sobre la identidad de los traductores, hay que reconocer la dificultad a la hora de encontrar información: concretamente en 21 casos no se ha podido encontrar ningún dato sobre el autor de la traducción. En cuanto a la valoración del trabajo del traductor, cuando existe, suele ser muy breve, y en cambio se puede decir que se prioriza el contenido del libro, importa más qué se ha traducido que cómo. Finalmente, sobre si el nombre del traductor contribuía a vender mejor la obra, pensamos que si el traductor era alguien conocido en el ámbito de la materia que traducía, o si el lector percibía una afinidad, sí podía influir en las ventas 
del libro; de ahí el interés del reseñador por mostrar esa afinidad en los casos en los que se producía.

Aunque se trate de textos en general breves, no hay duda de que los anuncios o las pequeñas reseñas de la sección de novedades bibliográficas de LIEA contribuyeron a la difusión de los últimos avances científicos y técnicos en nuestro país. Por otra parte, con su trabajo los traductores, además de acercar al lector las obras extranjeras, creaban al mismo tiempo la necesidad de elaborar obras especializadas en los distintos campos del conocimiento, contribuyendo así, con el uso de nuevos términos, al enriquecimiento de la propia lengua.

\section{Bibliografía}

Aymes, J.-R. 2010. La literatura francesa en La Ilustración Española y Americana (1888-1898). En Giné, M. \& Hibbs, S. (eds.) Traducción y cultura. Berna: Peter Lang, 87-106.

Botrel, J.-F. 2010. La literatura traducida: ¿es española? En Giné, M. \& Hibbs, S. (eds.) Traducción y cultura. Berna: Peter Lang, 27-40.

Corredor Plaja, A.-M. 2012. Presencia de la traducción en las novedades bibliográficas de La Ilustración Española y Americana (1869-1880). En Martino Alba, P. \& Jarilla, S. M. (eds.) Caleidoscopio de traducción literaria. Madrid: Dykinson, 25-43.

Corredor Plaja, A.-M. 2013. La difusión del saber: análisis e interpretación de la sección de novedades bibliográficas. En Giné, M.; Palenque, M. \& Goñi, J. M. (eds.) La recepción de la cultura extranjera en La Ilustración Española y Americana. Berna: Peter Lang, 113-133.

Palenque, M. 2013. La Ilustración Española y Americana, una ventana abierta a la cultura dominante en España entre 1869 y 1905. En Giné, M.; Palenque, M. \& Goñi, J. M. (eds.) La recepción de la cultura extranjera en La Ilustración Española y Americana. Berna: Peter Lang, 11-18.

Pinilla, J. \& Lépinette, B. 2009. La aportación propia del traductor al texto científico-técnico traducido o el afán de divulgación de un saber foráneo. A propósito del paratexto en una traducción al español de H. L. Duhamel du Monceau (1700-1782). Cuadernos del Instituto Historia de la Lengua 3: $109-125$.

Zaro, J. J. 2008. Diez estudios sobre la traducción en la España del siglo XIX. Granada: Atrio. 
